\title{
FECUNDITY AND SURVIVAL IN RELATION TO RESISTANCE TO OXIDATIVE STRESS IN A FREE-LIVING BIRD
}

\author{
Pierre Bize,${ }^{1,4}$ Godefroy Devevey, ${ }^{2}$ Patricia Monaghan, ${ }^{1}$ Blandine Doligez, ${ }^{3}$ and Philippe Christe ${ }^{2}$ \\ ${ }^{1}$ Division of Environmental and Evolutionary Biology, Glasgow University, Graham Kerr Building, Glasgow G20 8QQ United Kingdom \\ ${ }^{2}$ Department of Ecology and Evolution, University of Lausanne, Biophore, 1015 Lausanne, Switzerland \\ ${ }^{3}$ Department of Biometry and Evolutionary Biology, CNRS UMR 5558, University Lyon I, Bâtiment Gregor Mendel, \\ 43 Boulevard du 11 Novembre 1918, 69622 Villeurbanne Cedex, France
}

\begin{abstract}
Major life history traits, such as fecundity and survival, have been consistently demonstrated to covary positively in nature, some individuals having more resources than others to allocate to all aspects of their life history. Yet, little is known about which resources (or state variables) may account for such covariation. Reactive oxygen species (ROS) are natural by-products of metabolism and, when ROS production exceeds antioxidant defenses, organisms are exposed to oxidative stress that can have deleterious effects on their fecundity and survival. Using a wild, long-lived bird, the Alpine Swift (Apus melba), we examined whether individual red cell resistance to oxidative stress covaried with fecundity and survival. We found that males that survived to the next breeding season tended to be more resistant to oxidative stress, and females with higher resistance to oxidative stress laid larger clutches. Furthermore, the eggs of females with low resistance to oxidative stress were less likely to hatch than those of females with high resistance to oxidative stress. By swapping entire clutches at clutch completion, we then demonstrated that hatching failure was related to the production of low-quality eggs by females with low resistance to oxidative stress, rather than to inadequate parental care during incubation. Although male and female resistance to oxidative stress covaried with age, the relationships among oxidative stress, survival, and fecundity occurred independently of chronological age. Overall, our study suggests that oxidative stress may play a significant role in shaping fecundity and survival in the wild. It further suggests that the nature of the covariation between resistance to oxidative stress and life history traits is sex specific, high resistance to oxidative stress covarying primarily with fecundity in females and with survival in males.
\end{abstract}

Key words: Alpine Swift; annual survival; antioxidant defenses; Apus melba; fecundity; fitness traits; life history; oxidative theory of aging; sex-specific effect.

\section{INTRODUCTION}

Understanding why and how particular individuals pass on a greater number of gene copies to the following generation is at the hub of evolutionary ecology. The greater lifetime reproductive success of some individuals over others is usually explained by positive covariation between major life history traits, such as fecundity and survival (Stearns 1992, Reid et al. 2003a, Weladji et al. 2006). Indeed, although within-individual investment of finite resources into reproduction comes at the expense of investment in survival (Stearns 1992), positive covariation among individuals between fecundity and survival can arise if some individuals have more resources than others to allocate to all aspects of their life history (Van Noordwijk and de Jong 1986). Yet, it is poorly understood which resources (or state variables)

Manuscript received 10 July 2007; revised 9 November 2007; accepted 29 November 2007; final version received 23 January 2008. Corresponding Editor: K. Wilson.

${ }^{4}$ Present address: Department of Ecology and Evolution, Biophore, University of Lausanne, 1015 Lausanne-Dorigny, Switzerland. E-mail: Pierre.Bize@unil.ch positively affect both fecundity and survival, and in turn underpin their positive covariation across individuals (Zera and Harshman 2001, Ricklefs and Wikelski 2002).

Evidence is growing that the capacity to withstand oxidative stress plays an important role in shaping individual fecundity and survival (Beckman and Ames 1998, Finkel and Holbrook 2000, Barja 2004). Oxidative stress occurs when the production of reactive oxygen species (ROS) exceeds the antioxidant defenses (Finkel and Holbrook 2000, Falnes et al. 2007). ROS are byproducts of respiratory chains and they represent $0.1-$ $4 \%$ of the oxygen molecules consumed during respiration (Cadenas and Davies 2000). ROS are unstable compounds that can damage biomolecules such as DNA, proteins, and lipids, and consequently impair gene expression, tissue functioning, and ultimately organism reproductive success and viability (Beckman and Ames 1998, Finkel and Holbrook 2000, Barja 2004). ROS can lower individual reproductive success through two mutually nonexclusive ways, namely via effects on germinal and somatic cells. They can directly damage the reproductive system, and in turn harm offspring, by lowering spermatozoa, oocyte, and embryo quality or 
the quality of the environment in which the embryo will develop (Agarwal et al. 2006). Alternatively, ROSinduced damage can impair the somatic system of the parents (Linton et al. 2001, Praticò 2002), lowering their physiological or physical capacity to care for offspring and withstand environmental challenges. In the long term, the accumulation of ROS-induced damage to the soma is believed to contribute to the gradual decline of all organ systems (i.e., aging) and death (Beckman and Ames 1998, Finkel and Holbrook 2000, Barja 2004). Accordingly, variation in susceptibility to oxidative stress could account for positive covariation between major life history traits, in particular survival and fecundity. Because reproduction is an energetically demanding activity that increases individual metabolic load (Nilsson 2002) and, as a consequence, ROS production, individuals with high levels of resistance to oxidative stress may be better able to bear the costs of reproduction. This hypothesis predicts that individual capacity to resist oxidative stress may be a prime factor underling variation in individual state and, in turn, reproductive success and survival, with those individuals having higher resistance to oxidative stress achieving a higher breeding performance and survival. Although previous laboratory studies on model species have confirmed the role played by ROS in shaping individual fecundity and survival (Beckman and Ames 1998, Finkel and Holbrook 2000, Barja 2004), we still know very little about the links between resistance to oxidative stress and reproductive success and survival in the wild. Experimental manipulation of dietary antioxidants in free-living animals has highlighted that these are often a limited resource in nature (e.g., Blount et al. 2004, Biard et al. 2005). Detailed studies on individual resistance to oxidative stress, reproduction, and survival are now required to validate the role played by oxidative stress in shaping the life history trajectories of free-living organisms.

In the present study, we used data from a colonial wild bird, the Alpine Swift (Apus melba), to investigate whether interindividual variation in fecundity (i.e., clutch size and egg hatchability) and annual survival is related to interindividual variation in red cell resistance to oxidative stress. Because the Alpine Swift is a longlived bird (the oldest recaptured individual reported thus far was 26 years of age; Swiss Ornithological Station, personal communication) and interindividual variation in oxidative stress can covary with age (e.g., Linton et al. 2001, Praticò 2002, Gil et al. 2006), interindividual variation in age may confound potential relationships among oxidative stress, fecundity, and annual survival. Therefore, performing analyses on known-age individuals is a first important step to ensure that interindividual variation in oxidative stress, rather than chronological age, is the biological factor accounting for interindividual variation in fecundity and survival (Johnson 2006). We applied this approach to our study population, where most of the individuals (74\%) are of known age. Thus, after controlling for age effects, we predicted that, if resistance to oxidative stress per se is central to sustain the (oxidative) cost of reproduction (Alonso-Alvarez et al. 2004, Wiersma et al. 2004, Bertrand et al. 2006), then resistant individuals will have higher fecundity and/or survival. Parental oxidative state could influence fecundity through effects on propagule (genetic or phenotypic) quality and/or parental care. In this study, we were able to examine clutch size and egg hatchability, two important determinants of reproductive success in the Alpine Swift (Arn 1960), and obtained accurate data on adult survival to the next breeding season. We experimentally separated parental care and propagule quality effects by swapping entire clutches between randomly chosen pairs of nests before the start of incubation. We predicted that, if parental state affects egg hatchability through the production of low-quality eggs, hatching success should be related to the capacity of biological, but not foster, parents to resist oxidative stress. Alternatively, if egg hatching success relies on the quality of parental care received during incubation, egg hatchability should be related to the resistance of foster, not biological, parents.

\section{Methods}

The Alpine Swift is a 90-g migratory bird that feeds on aerial insects. It breeds in colonies varying in size from a few $(<5)$ up to several hundred pairs located in holes in cliffs or under the roofs of tall buildings. It forms socially monogamous breeding pairs that are usually maintained over several years (Arn 1960). A single clutch of 1-4 eggs is produced per year (modal clutch size is three eggs). Both parents incubate the clutch for 20 days and feed the offspring up to fledging, which occurs 50-70 days after hatching (Bize et al. 2004).

Fieldwork was carried out in 2005 and 2006 in Switzerland in two Alpine Swift colonies located in Bienne $(\sim 100$ breeding pairs $)$ and Solothurn $(\sim 50$ breeding pairs). Because Alpine Swift fledglings are philopatric and return to breed in their natal colony (Arn 1960), and because all of the fledglings were individually recognized using numbered aluminum bands in these two study colonies during the past 40 years, most of the adults $(74 \%)$ recruited in these two colonies are of known age. In 2005, the age of breeders was $5.85 \pm 0.27$ years, mean $\pm \mathrm{SE}$ (range $2-18$ years), and did not differ between the two colonies (Student $t$ test: $t=0.18$, $\mathrm{df}=161, P=0.86$ ). In both colonies, virtually all of the breeders are recaptured each year (capture-recapture models show that recapture rate of breeders is $1.0 \pm 0.003$, mean $\pm \mathrm{SE}$; Bize et al. 2006). Hence, annual return rate of breeders provides a good measure of annual survival.

In both years, nests were visited daily to determine laying date, clutch size, and brood size at hatching. Adults were captured while sitting on eggs or hatchlings, and if they had not already been banded as nestlings, 
they were banded at first capture when adults. The same person (P. Bize) weighed all adults to the nearest $0.1 \mathrm{~g}$, and measured the length of the sternum to the nearest $0.1 \mathrm{~mm}$ and of the left wing to the nearest $1.0 \mathrm{~mm}$. For each adult, as an index of body size, we calculated the first principal component (PC1) of a principal component analysis (PCA), with body mass, sternum length, and wing length as loading factors (eigenvectors: body mass $=0.55$, sternum length $=0.60$, wing length $=0.58$ ) A high PC1 index indicates large and heavy individuals. As an index of body condition, we used the second component (PC2) of the same PCA (eigenvectors: body mass $=0.81$, sternum length $=-0.22$, wing length $=$ $-0.55)$. The PC2 index is strongly positively correlated with the residuals of a linear regression of body mass on sternum length and wing length (Pearson correlation: $r=$ $0.87, n=219$ birds, $P<0.001$ ); thus a high PC2 index indicates individuals with high energy reserves (Ardia 2005). A blood sample $(\sim 150 \mu \mathrm{L})$ was collected from the foot of each adult using heparinized capillaries to measure the resistance of red blood cells to oxidative stress, and to sex the birds using blood cell DNA and molecular techniques (Bize et al. 2005).

\section{Assessment of red blood cell resistance to oxidative stress}

We measured resistance to oxidative stress as the time needed to hemolyze $50 \%$ of the red blood cells exposed to a controlled attack by ROS using the KRL bioassay (Brevet Spiral V02023, Courernon, France). A quick lyse of red cells indicates low resistance of the red cell membrane to ROS attack. Resistance of the red blood cell membrane to ROS attack is shaped by membrane lipid composition and past exposure of unsaturated lipid bonds to oxidative stress (Brzezinska-Slebodzinska 2001) as well as by levels of intra- and extracellular antioxidant defenses (i.e., antioxidant compounds and enzymes) against ROS (Lesgards et al. 2002). Thus, our measurement of resistance to oxidative stress is likely to be influenced by genetic factors (i.e., membrane composition and antioxidant enzymes) and by environmental factors (i.e., dietary antioxidants and past exposure of the cell membrane to ROS). The high resistance of red blood cell to oxidative stress of some individuals compared to others can therefore reflect aspects of individual genetic quality and/or phenotypic quality.

To measure red blood cell resistance to oxidative stress, $16 \mu \mathrm{L}$ of overall blood were immediately diluted in $584 \mu \mathrm{L}$ of $\mathrm{KRL}$ buffer adjusted to avian cell osmolarity. Samples were stored at $4^{\circ} \mathrm{C}$ in the field before analysis in the laboratory, which occurred $24.7 \pm$ $0.7 \mathrm{~h}$ (mean $\pm \mathrm{SE}$; range $2.5-54 \mathrm{~h}$ ) after blood collection. We incubated $90 \mu \mathrm{L}$ of KRL-diluted overall blood at $40^{\circ} \mathrm{C}$ and submitted it to a controlled ROS attack by adding a solution of 150 millimoles of $2,2^{\prime}$ azobis-(aminodinopropane) hydrochloride diluted in $153 \mu \mathrm{L}$ of KRL buffer. The time needed to lyse $50 \%$ of the red blood cells was assessed with a microplate reader device that follows the decrease of optical density at the wavelength of $540 \mathrm{~nm}$. Samples were run in triplicates (intra-plate repeatability: $r=0.986, P<$ 0.0001 ), and mean values were calculated for the analyses. Red blood cell resistance to oxidative stress significantly and linearly decreased with increasing time interval between blood sampling and the exposure of red blood cells to $\operatorname{ROS}\left(F_{1,217}=23.40, P<0.0001\right)$. Hence, we standardized our measure of resistance to oxidative stress by using the residuals of a linear regression of observed resistance to oxidative stress on the time interval between blood sampling and exposure of red blood cells to ROS. In total, we measured resistance of red blood cells to oxidative stress (hereafter, abbreviated throughout the text as "resistance to oxidative stress") in 219 breeding individuals, of which 163 (83 females and 80 males) were of known age.

\section{Cross-fostering experiment}

In 2005 in the colony in Solothurn, we swapped complete clutches between 19 pairs of nests within 48 hours after clutch completion. Clutches were matched for laying date and clutch size. Three out of the 38 clutches were abandoned by parents during incubation, most probably in response to poor weather conditions prevailing during the summer of 2005, and they were assigned a zero hatching success. The exclusion of these three clutches from the analyses does not qualitatively change our results. We were able to measure resistance to oxidative stress of 34 out of 38 experimental mothers and 35 out of 38 experimental fathers. There was no correlation in resistance to oxidative stress of foster and biological fathers (Pearson correlation: $r=-0.05, P=$ $0.85, n=16$ ). By chance, however, resistance to oxidative stress was positively and significantly correlated between foster and biological mothers (Pearson correlation: $r=$ $0.58, P=0.023, n=15)$. Although this somewhat reduces our ability to examine the independent effects of the biological and foster mothers, there is still a substantial amount of unexplained variation in the relationship between the two variables. The cross-fostering experiment and blood sampling were carried out under veterinary licenses from the Cantons Berne (BE 56/05) and Solothurn (SO 02/06).

\section{Statistical analyses}

Data were analyzed using generalized linear mixed models in SAS (PROC GLIMMIX in SAS version 9.1; SAS Institute, Cary, North Carolina, USA), allowing us to specify the appropriate error structure. Interindividual variation in resistance to oxidative stress was analyzed with a normal error, clutch size with a lognormal error, and egg hatchability (i.e., number of hatched eggs/clutch size) and returning rate (i.e., coded $1 / 0$ if the bird returned/did not return to the colony, respectively) with a binomial error structure and logitlink function. In the observational data collected in Bienne and Solothurn, we controlled for the effect of colony on adult resistance to oxidative stress, clutch size, 
TABLE 1. Results of mixed models for Alpine Swifts (Apus $m e l b a$ ), with male and female data pooled to investigate sexspecific effects (i.e., interaction) between (a) (age $)^{2}$ and resistance to oxidative stress, and between resistance to oxidative stress and (b) clutch size, (c) egg hatchability, and (d) annual survival.

\begin{tabular}{lcrc}
\hline \hline \multicolumn{1}{c}{ Source of variation } & df & $F$ & $P$ \\
\hline a) Resistance to ROS & & & \\
Sex & 1,116 & 1.04 & 0.31 \\
Age & 1,156 & 11.49 & $<0.001$ \\
$(\text { Age })^{2}$ & 1,158 & 8.88 & 0.003 \\
Sex $\times(\text { Age })^{2}$ & 1,132 & 8.41 & 0.004 \\
b) Clutch size & & & \\
Sex & 1,157 & 0.02 & 0.88 \\
Resistance to ROS & 1,157 & 2.12 & 0.15 \\
Age & 1,157 & 12.28 & $<0.001$ \\
Age) & 1,157 & 5.04 & 0.026 \\
Sex $\times$ Resistance to ROS & 1,157 & 3.95 & 0.048 \\
c) Egg hatchability & & & \\
Sex & 1,149 & 0.00 & 0.97 \\
Resistance to ROS & 1,149 & 3.97 & 0.048 \\
Sex $\times$ Resistance to ROS & 1,149 & 0.79 & 0.38 \\
d) Annual survival & & & \\
Sex & 1,159 & 2.15 & 0.14 \\
Resistance to ROS & 1,159 & 0.16 & 0.69 \\
Sex $\times$ Resistance to ROS & 1,159 & 4.75 & 0.031 \\
\end{tabular}

Notes: Effect of age and (age $)^{2}$ did not explain a significant part of the variance in models (c) and (d), and thus these two factors were dropped from the final models. Reactive oxygen species (ROS) are natural by-products of metabolism, and when ROS production exceeds antioxidant defenses, organisms are exposed to oxidative stress.

egg hatchability, and annual survival by including the colony as a random variable in the mixed models. When analyzing egg hatchability, we controlled for potential local microenvironmental effects by including the sector of the colony within which the nest was located as a second random variable. We entered age and (age $)^{2}$ as two covariates in our starting models to account for nonlinear age-related variation. We performed separate analyses for male and female birds to account for sexspecific variation in resistance to oxidative stress, fecundity, and survival. To explicitly test the interaction between sex and the focal covariate, we also ran mixed models with pooled data from both sexes and with the breeding pair identity included as a random variable to control for the fact that both parents of a given nest may have been measured ( $n=50$ pairs for which age and resistance to oxidative stress of both partners were known). Analyses were conducted with a full model; nonsignificant terms, starting with nonsignificant interactions, were then backward dropped until the single best final model was found. All tests were two tailed and $P<0.05$ was considered significant. Satterthwaite correction was used to approximate the degree of freedom for fixed effects. Due to ongoing experiments at the nestling stage, we did not analyze covariation between parent resistance to oxidative stress and fledging success. Note, however, that swifts lay small clutches (mean 2.6 eggs per clutch), and therefore hatching failure has large effect on fledging success. The number of young at fledging is correlated with the number of young at hatching (Pearson correlation: $r=$ $0.65, P<0.0001, n=2168$ breeding attempts recorded by Arn [1960] between 1932 and 1956 in Solothurn).

\section{Results}

\section{Interindividual variation in resistance to oxidative stress}

Female resistance to oxidative stress increased between 2 and 4 years of age and reached an asymptote between 5 and 10 years of age before decreasing at older ages (significant effect of age and (age) ${ }^{2}$ in Tables $1 \mathrm{a}$ and 2a, Fig. 1). Conversely, male resistance to oxidative stress increased linearly with age (significant effect of age in Tables 1a and 2a, Fig. 1) and showed no significant decline at older ages, even though the data for males extended to older ages than for females. Female and male variation in resistance to oxidative stress was not explained by variation in adult body size (PC1) and body condition (PC2) (Table 2a). Additional analyses in which body mass, wing length, and sternum length were entered in the models instead of PC1 and PC2 provided similar results (all $P$ values $>0.69$ ), further emphasizing that resistance to oxidative stress is not linked to size or condition. Sex-specific variation in resistance to oxidative stress at an older age is further demonstrated by the significant interaction between sex and (age) ${ }^{2}$ when both sexes were tested together (Table 1a). This model also showed that, on average, red blood cells of males and females were similarly resistant to oxidative stress (least square [LS] means of male vs. female resistance to oxidative stress: $0.48 \pm 0.67$ vs. $-0.95 \pm 0.67$, mean \pm $\mathrm{SE}$; effect of sex is not significant in Table 2a). Pairing was assortative with respect to resistance to oxidative stress (Pearson correlation between male and female resistance to oxidative stress, controlling for age of both partners: $r=0.31, P=0.028, n=50$ pairs for which both partners were of known age; Fig. 2), and random with respect to body size $(r=0.01, P=0.97, n=50)$ and body condition $(r=-0.01, P=0.99, n=50)$.

\section{Resistance to oxidative stress and fecundity}

Females that had higher resistance to oxidative stress laid larger clutches, independently of the effect of age on clutch size (Tables $1 \mathrm{~b}$ and 2b, Fig. 3). In males, clutch size significantly increased with age, but not with resistance to oxidative stress (Table 2b, Fig. 3). This sex-specific relationship between resistance to oxidative stress and clutch size is further confirmed by the significant interaction between sex and resistance to oxidative stress when both sexes were tested together (Table 1b). The exclusion from the analyses of the single female that laid a clutch of four eggs (Fig. 3) does not change our results.

The cross-fostering experiment of entire clutches at the onset of incubation revealed that egg hatchability was significantly related to resistance to oxidative stress of biological mothers, but not biological fathers, foster 
TABLE 2. Results of separate mixed models for males and females for (a) resistance to oxidative stress in relation to age and indices of body condition and body size, and (b) clutch size, (c) egg hatchability, and (d) adult annual survival in relation to resistance to oxidative stress and age.

\begin{tabular}{|c|c|c|c|c|c|c|}
\hline \multirow[b]{2}{*}{ Source of variation } & \multicolumn{3}{|c|}{ Females } & \multicolumn{3}{|c|}{ Males } \\
\hline & df & $F$ & $P$ & df & $F$ & $P$ \\
\hline \multicolumn{7}{|l|}{ a) Resistance to ROS } \\
\hline Age & $1,71.1$ & 8.96 & 0.004 & 1,78 & 11.56 & 0.001 \\
\hline$(\text { Age })^{2}$ & $1,78.8$ & 8.70 & 0.004 & 1,77 & 1.36 & 0.25 \\
\hline $\mathrm{PCl}$ (size) & $1,78.8$ & 0.21 & 0.65 & 1,76 & 0.07 & 0.79 \\
\hline PC2 (condition) & $1,77.8$ & 0.06 & 0.81 & 1,75 & 0.02 & 0.89 \\
\hline \multicolumn{7}{|l|}{ b) Clutch size } \\
\hline Resistance to ROS & 1,80 & 8.99 & 0.004 & 1,77 & 0.01 & 0.92 \\
\hline Age & 1,80 & 21.56 & 0.001 & 1,78 & 5.86 & 0.02 \\
\hline$(\text { Age })^{2}$ & 1,79 & 3.94 & 0.06 & 1,76 & 0.41 & 0.52 \\
\hline \multicolumn{7}{|l|}{ c) Egg hatchability } \\
\hline Resistance to ROS & 1,75 & 4.39 & 0.04 & 1,74 & 0.36 & 0.55 \\
\hline Age & 1,74 & 2.41 & 0.12 & 1,73 & 0.00 & 0.97 \\
\hline$(\text { Age })^{2}$ & 1,73 & 3.37 & 0.07 & 1,72 & 0.09 & 0.76 \\
\hline \multicolumn{7}{|l|}{ d) Annual survival } \\
\hline Resistance to ROS & 1,81 & 1.71 & 0.19 & 1,78 & 3.00 & 0.09 \\
\hline Age & 1,80 & 0.09 & 0.77 & 1,77 & 0.14 & 0.71 \\
\hline$(\text { Age })^{2}$ & 1,79 & 0.45 & 0.51 & 1,76 & 1.13 & 0.29 \\
\hline
\end{tabular}

Note: Significant terms are reported in boldface; nonsignificant terms, starting with nonsignificant interactions, were backwarddropped from the models.

mothers, or foster fathers (Table 3). This finding was backed up by analyses on the probability of eggs hatching in pooled experimental and nonexperimental clutches (Solothurn and Bienne, respectively), showing that egg hatchability was explained by resistance to oxidative stress of biological mothers (Table 2c, Fig. 4), but not biological fathers (Table 2c, Fig. 4). When both sexes were tested together, the interaction between sex and resistance to oxidative stress was not significant (Table 1c), which may come from the fact that female resistance to oxidative stress had only a weak, but significant, effect ( $P=0.04$; Table 2c) on egg hatchability.

\section{Resistance to oxidative stress and annual survival}

The relationship between resistance to oxidative stress and annual survival differed between the sexes, as shown by the significant interaction between resistance to oxidative stress and sex in Table 1d. This interaction can be explained by the fact that males with higher resistance to oxidative stress tended to be more likely to survive to the following year $(P=0.09$; Table $2 \mathrm{~d})$,
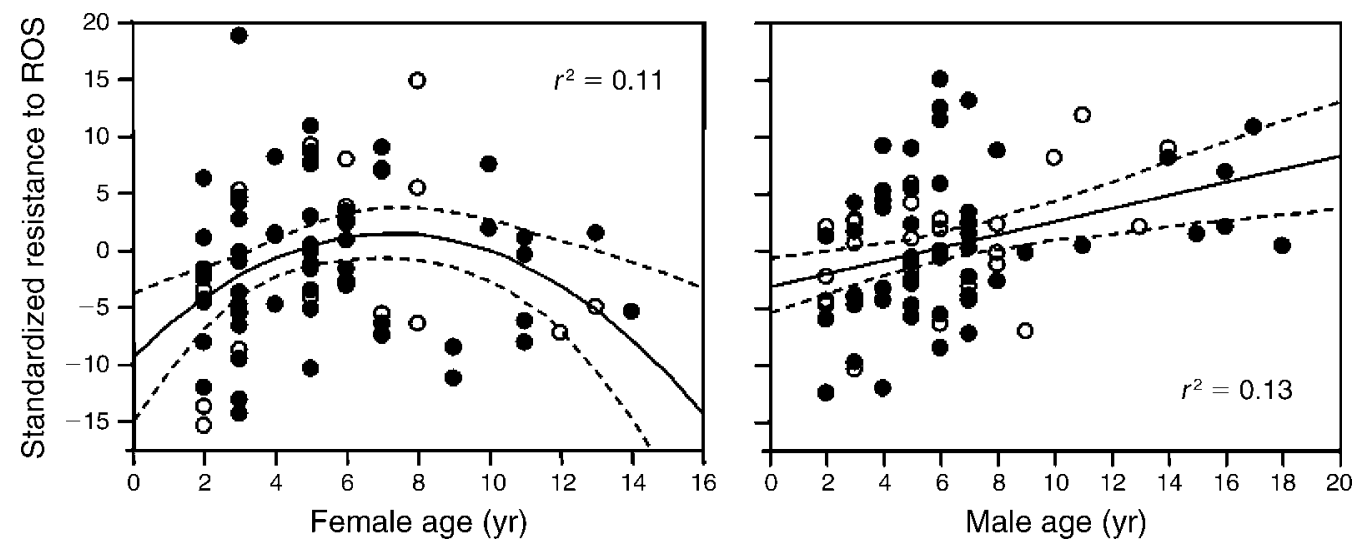

FIG. 1. Age-related variation in standardized resistance to oxidative stress in female and male Alpine Swifts (Apus melba) in the colonies Bienne and Solothurn, Switzerland (solid and open symbols, respectively). When metabolic production of reactive oxygen species (ROS) exceeds antioxidant defenses, organisms are exposed to oxidative stress. Resistance to oxidative stress is measured as the time needed to hemolyze $50 \%$ of the red blood cells exposed to a controlled attack by ROS. Those measurements were performed in the laboratory within $54 \mathrm{~h}$ after blood sampling and were standardized by using the residuals of a linear regression of observed resistance to oxidative stress on the time interval between blood sampling and exposure of red blood cells to ROS. Fitted lines are shown with $95 \%$ confidence intervals (dashed lines); the proportion of the variance around the mean explained by the fitted lines $\left(r^{2}\right)$ is reported in the figure. $N=63$ and 20 females and 52 and 28 males in Bienne and Solothurn, respectively. 


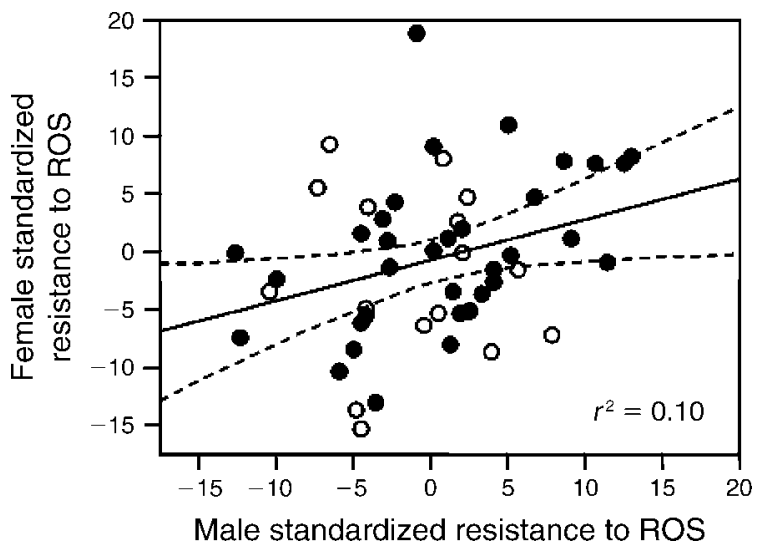

FIG. 2. Assortative mating with respect to standardized resistance to oxidative stress in the colonies Bienne and Solothurn (solid and open symbols, respectively). Each dot represents the resistance of each member of a mated pair. The fitted line is shown with $95 \%$ confidence intervals (dashed lines); the proportion of the variance around the mean explained by the regression line $\left(r^{2}\right)$ is reported in the figure. $N=34$ and 16 breeding pairs in Bienne and Solothurn, respectively.

whereas resistance to oxidative stress did not explain variation in female annual survival $(P=0.19$; Table 2d). Additional analyses in which resistance to oxidative stress was entered as the dependent variable (rather than as the explanatory variable) and survival was entered as the explanatory variable (rather than as the dependent variable) showed that males that survive to the following year were significantly less susceptible to oxidative stress ( $t$ test: $t=1.75, \mathrm{df}=78, P=0.042$ ), whereas no difference was found in females $(t=-1.31, \mathrm{df}=81, P=0.19)$ (Fig. 5).

\section{DisCuSSION}

In agreement with the hypothesis that oxidative stress incurs costs to the organisms in terms of reduced fecundity and survival (Beckman and Ames 1998, Finkel and Holbrook 2000, Barja 2004), Alpine Swift females with lower resistance to oxidative stress laid clutches that were smaller and less likely to hatch than did females with higher resistance to oxidative stress, and males that survived up to the next breeding season were more resistant to oxidative stress. Although male and female resistance to oxidative stress covaried with age, our findings were not confounded by older individuals becoming less resistant to oxidative stress (Linton et al. 2001, Praticò 2002, Gil et al. 2006), less fecund, and having lower survival (e.g., Broussard et al. 2003, Reid et al. 2003b). Here, note that the relationships between male resistance to oxidative stress and survival were not particularly robust $(P=0.04$ and 0.09$)$, and thus it will be important to replicate this result with larger sample sizes collected over several years. Hereafter, we discuss in more detail how susceptibility to oxidative stress can shape individual fecundity and survival, and hence what information resistance to oxidative stress may provide about the biological state of an individual and, in turn, about its life history trajectory.

\section{Resistance to oxidative stress and fitness}

Previous experimental studies in birds in which females were supplemented with dietary antioxidants have demonstrated that antioxidant availability and, in turn, resistance to oxidative stress, can limit egg-laying capacity (Blount et al. 2004, Sahin et al. 2006) and egg hatchability (McGraw et al. 2005, Pappas et al. 2006). Increased fecundity in females through the consumption of dietary antioxidants has also been documented in sea urchins (George et al. 2001), fish (Shahidi et al. 1998, Pettersson and Lignell 1999), and mammals (Chew and Archer 1983, Brief and Chew 1985). In agreement with these studies, we found that Alpine Swift females that were more resistant to oxidative stress were also more fecund (measured in terms of egg production and egg hatchability). Yet, the exact mechanisms linking resistance to oxidative stress to fecundity are unknown and remain to be investigated. Two main routes can be
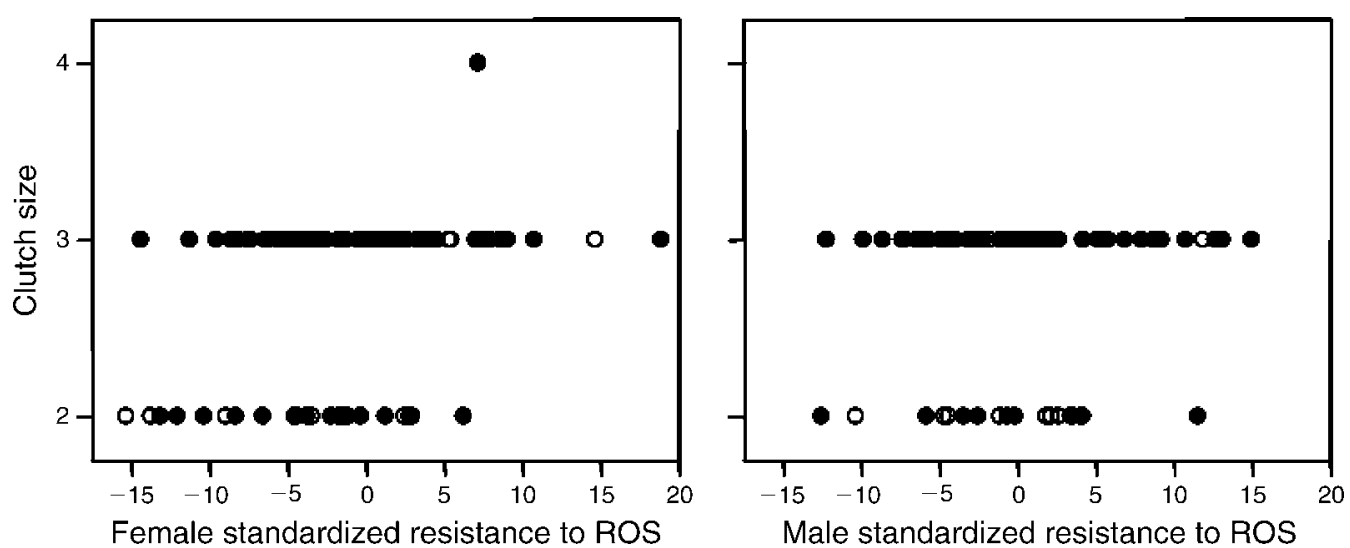

FIG. 3. Clutch size in relation to standardized resistance to oxidative stress in female and male Alpine Swifts in the colonies Bienne and Solothurn (solid and open symbols, respectively). $N=63$ and 20 females and 52 and 28 males in Bienne and Solothurn, respectively. 
TABLE 3. Relationship between egg hatchability and resistance to oxidative stress of biological and foster parents in experimentally cross-fostered clutches ( $n=38$ clutches) in the colony Solothurn.

\begin{tabular}{lcrl}
\hline \hline \multicolumn{1}{c}{ Source of variation } & df & \multicolumn{1}{c}{$F$} & $P$ \\
\hline Resistance to ROS of biological mother & 1,32 & 13.01 & 0.001 \\
Resistance to ROS of biological father & 1,28 & 1.54 & 0.23 \\
Resistance to ROS of foster mother & 1,27 & 0.11 & 0.74 \\
Resistance to ROS of foster father & 1,28 & 0.35 & 0.56
\end{tabular}

Notes: Stepwise backward models showed that only resistance to oxidative stress of biological mothers explains egg hatchability. Effects of nonsignificant variables tested along with resistance to oxidative stress of biological mothers are reported.

suggested. First, resistance to oxidative stress may confer reproductive benefits by conferring protection to the somatic system against ROS (Linton et al. 2001, Praticò 2002). This hypothesis predicts that individuals with higher resistance to oxidative stress are in better condition and thus are able to allocate more resources to reproduction. However, in our case, females (and males) with higher resistance to oxidative stress were not obviously larger or in better condition than individuals with lower resistance oxidative stress. In the crossfostering experiment, egg hatchability also was not explained by resistance of foster parents to oxidative stress and, by extension, by the ability of foster parents to care for eggs during incubation. Second, resistance to oxidative stress may increase reproductive success by conferring protection to the reproductive system against ROS. Evidence is growing in the human reproductive biology literature that ROS can modulate the maturation and functioning of female and male reproductive system and, in turn, the production and viability of spermatozoa, oocytes, and embryos (Agarwal et al. 2006). By cross-fostering complete clutches before the start of incubation, we showed that egg hatchability was explained by resistance to oxidative stress of the biological mothers. Because our measurement of resis- tance to oxidative stress can reflect aspects of the phenotypic quality of individuals (e.g., circulating levels of dietary antioxidants; Lesgards et al. 2002) and/or genetic quality (e.g., cell membrane composition and integrity; Brzezinska-Slebodzinska 2001), the positive relationship between female resistance to oxidative stress and egg hatchability could be a consequence of environmental and/or genetic effects. That is, females with high resistance to oxidative stress might have, for instance, greater amounts of antioxidants available to transfer to their offspring via the egg yolk (i.e., maternal effects; McGraw et al. 2005, Pappas et al. 2006). Alternatively, females with high resistance to oxidative stress may be genetically less susceptible to oxidative stress and, in turn, may produce offspring of similarly high genetic quality. Although ROS can also affect the male reproductive system (Agarwal et al. 2006), we found no clear evidence that egg hatchability is related to male resistance to oxidative stress. This lack of evidence may come from the anisogamy in gamete size and number, which reduces the chance of detecting such effects in males compared to females. Thus, future studies of males should be focused on sperm quality rather than on egg hatchability to test the link between antioxidant defenses and fecundity.

The effects of ROS on life span have been intensively examined in laboratory studies on nematodes (Caenorhabitis elegans; Ishii et al. 1998), fruit flies (Drosophila melanogaster; Parkes et al. 1998, Ruan et al. 2002), and mice (Mus musculus; Moskovitz et al. 2001, Holzenberger et al. 2003). In agreement with the free radical theory of aging which states that oxidative damage accumulates throughout life and contributes to aging and death (Beckman and Ames 1998, Finkel and Holbrook 2000, Barja 2004), these studies have repeatedly demonstrated that the ability of model organisms to resist oxidative stress plays a central role in life span extension (for similar results on a non-model organism, see Pike et al. 2007). Although we did not measure life span, we found that annual survival tended $(P=0.04$

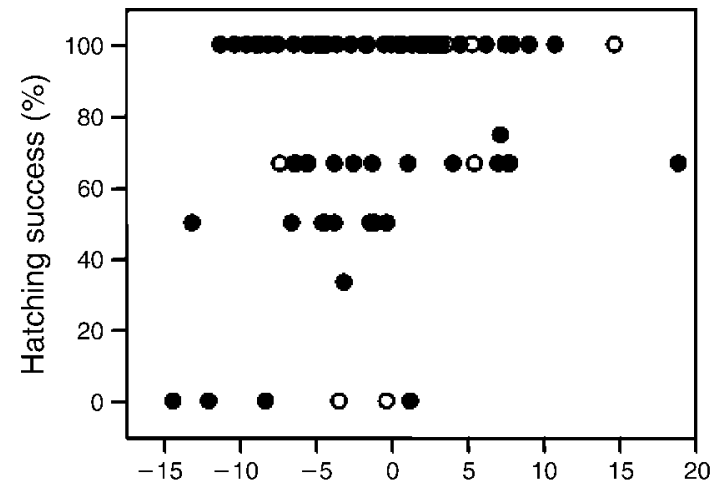

Female standardized resistance to ROS

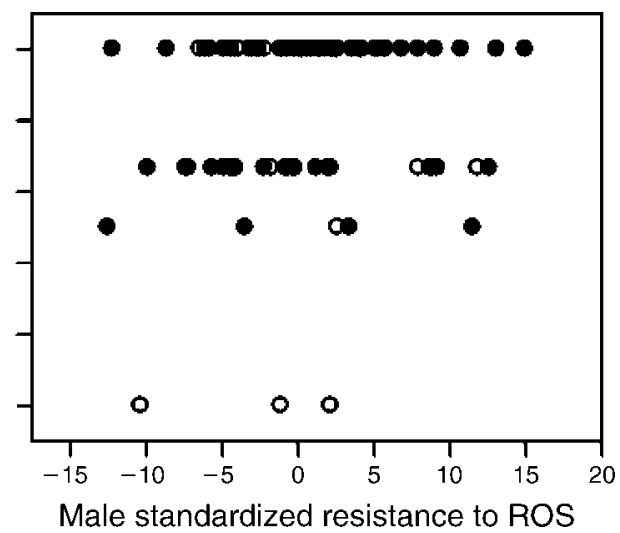

Male standardized resistance to ROS

FIG. 4. Hatching success in relation to standardized resistance to oxidative stress in female and male Alpine Swifts in the colonies Bienne and Solothurn (solid and open symbols, respectively). $N=63$ and 20 females and 52 and 28 males in Bienne and Solothurn, respectively. 
FIG. 5. Resistance to oxidative stress (mean $\pm \mathrm{SE}$ ) in female and male Alpine Swifts as a function of whether they returned or not in the following year to their breeding colonies in Bienne and Solothurn (i.e., index of annual survival). Sample sizes are given above the histogram bars.
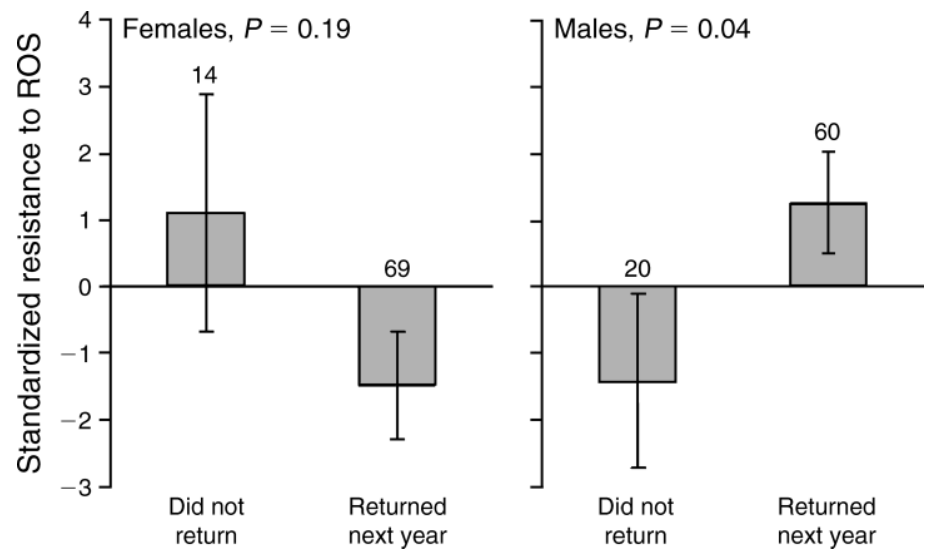

and 0.09) to be positively related to resistance to oxidative stress in Alpine Swift males only. Our results are in agreement with a recent study on captive Zebra Finches (Taeniopygia guttata) showing that resistance of red blood cells to oxidative stress predicted individual short-term (within three months) survival (AlonsoAlvarez et al. 2006). As for fecundity, the proximate mechanisms linking resistance to oxidative stress to survival remain to be investigated. Although numerous pathways have been proposed (and currently are being investigated and debated in the literature; reviewed in Beckman and Ames 1998, Finkel and Holbrook 2000, Barja 2004), and although evidence is increasing that life span regulation by ROS is a multifactorial process, the dominant mechanisms remain unclear. One problem may come from the fact that a large number of the candidate mechanisms have been studied in gene knockout individuals (with genes removed to test function) that are unlikely to survive (evolve) in natural environments (Leslie 2001). In the present context, the Alpine Swift may be a valuable study system in which to pinpoint dominant pathways producing the link between oxidative stress and survival in nature. We are currently investigating the relationships among oxidative stress, telomere dynamics, and life span (Monaghan and Haussmann 2006) in this bird species.

\section{Resistance to oxidative stress as a biomarker of individual state and life history trajectory}

Heterogeneity in individual fitness is generally explained by state-dependent mechanisms, with individuals of different states being more or less able to sustain the costs of reproduction (McNamara and Houston 1996). In iteroparous organisms, breeder age is a wellknown state variable, with middle-aged individuals usually achieving a higher reproductive success and survival than young and/or old individuals (e.g., Broussard et al. 2003, Reid et al. 2003b). By definition, however, age is a chronological parameter that provides little information about the biological factors mediating variation in individual fitness (Johnson 2006). In the present study, we found that resistance of red blood cells to oxidative stress covaried with age in female and male Alpine Swifts, but the relationships between oxidative stress, female fecundity, and male survival occurred independently of chronological age. Hence, our findings suggest that red blood cell resistance to oxidative stress may provide a valuable biomarker of individual state.

The identification of biomarkers of individual state may provide important tools to monitor the processes that underlie variation in life history trajectories (Ricklefs and Wikelski 2002, Monaghan and Haussmann 2006). Here, for instance, resistance to oxidative stress may give important insights into the constraints imposed by aerobic metabolism on the diversification of life histories. Accordingly, a recent study of Zebra Finches in which offspring were raised either in reduced or enlarged broods showed that early growth condition had effects on a suite of life history traits, including age at first reproduction, fecundity, and longevity, and that optimal allocation of resources between reproduction and resistance to oxidative stress could be the underlying modulatory mechanism (Alonso-Alvarez et al. 2006). Indeed, brood size manipulation affected offspring red cell resistance to oxidative stress, and heterogeneity in offspring reproductive performance and survival was significantly related to variation in resistance to oxidative stress (Alonso-Alvarez et al. 2006). In our study, we found that the relationships among resistance to oxidative stress, age, fecundity, and survival were sex specific: females with high resistance to oxidative stress had a higher fecundity, and males with high resistance to oxidative stress tended to have a higher annual survival. This is in line with the Bateman principle, which suggests that life history strategies are sex specific (Bateman 1948); namely, that female fitness is determined by access to resources and fecundity and male fitness is determined by access to mates and longevity. Interestingly, sex-specific variation in resistance to oxidative stress has also been reported in adult Zebra Finches, with males being more susceptible to oxidative stress than females when forced to work harder (AlonsoAlvarez et al. 2004, Wiersma et al. 2004). One striking difference between male and female Alpine Swifts was in 
age-related variation in resistance to oxidative stress, with resistance to oxidative stress declining at older ages in females and increasing linearly with age in males. Because our results are based on cross-sectional data rather than on longitudinal data, it is unclear to what extent this sex-specific variation in resistance to oxidative stress is due to within-individual change (senescence in females and improvement in males) and between individual differences (selective appearance and disappearance) (van de Pol and Verhulst 2006). To solve this problem, long-term data in which the same individuals are repeatedly measured throughout their lives are required. The fact that males that were less resistant to oxidative stress were less likely to return to the colonies in the following year suggests, nonetheless, that the increase in resistance to oxidative stress with age in males may result form the selective disappearance in the population of those males less resistant to oxidative stress rather than to within-individual improvement with age. Further experimental, longitudinal, and comparative studies using a wide range of organisms are now needed for a better appraisal of the role played by oxidative stress in the diversification of life histories. Because oxidative stress comes from an imbalance between antioxidant defenses and ROS production, one important challenge will be to evaluate the relative contribution of variation in antioxidant defenses and variation in ROS production in driving the diversification of life histories (Criscuolo et al. 2005).

\section{ACKNOWLEDGMENTS}

We are grateful to Caroline Jeanneret, Aurélie Klopfenstein, Antoine Stocker, and Candice Yvon for assistance in the field, Dominik Pfluger and the late Theo Marbot for allowing us access to the Alpine Swift colonies in Solothurn and Bienne, respectively, and to the Swiss National Science Foundation for financial support (grant number PP00A-109009 to P. Bize).

\section{Literature Cited}

Agarwal, A., S. Gupta, and S. Sikka. 2006. The role of free radicals and antioxidants in reproduction. Current Opinion in Obstetrics and Gynecology 18:325-332.

Alonso-Alvarez, C., S. Bertrand, G. Devevey, J. Prost, B. Faivre, O. Chastel, and G. Sorci. 2006. An experimental manipulation of life-history trajectories and resistance to oxidative stress. Evolution 60:1913-1924.

Alonso-Alvarez, C., S. Bertrand, G. Devevey, J. Prost, B. Faivre, and G. Sorci. 2004. Increased susceptibility to oxidative stress as a proximate cost of reproduction. Ecology Letters 7:363-368.

Ardia, D. R. 2005. Super size me: an experimental test of the factors affecting lipid content and the ability of residual body mass to predict lipid stores in nestling European Starlings. Functional Ecology 19:414-420.

Arn, H. 1960. Biologische Studien am Alpensegler. Verlag Vogt-Schild AG, Solothurn, Switzerland.

Barja, G. 2004. Free radicals and aging. Trends in Neurosciences 27:595-600.

Bateman, A. J. 1948. Intra-sexual selection in Drosophila. Heredity 2:349-368.

Beckman, K. B., and B. N. Ames. 1998. The free radical theory of aging matures. Physiological Reviews 78:547-581.

Bertrand, S., C. Alonso-Alvarez, G. Devevey, B. Faivre, J. Prost, and G. Sorci. 2006. Carotenoids modulate the trade- off between egg production and resistance to oxidative stress in zebra finches. Oecologia 147:576-584.

Biard, C., P. F. Surai, and A. P. Møller. 2005. Effects of carotenoid availability during laying on reproduction in the blue tit. Oecologia 144:32-44.

Bize, P., J. Gasparini, A. Klopfenstein, R. Altwegg, and A. Roulin. 2006. Melanin-based coloration is a nondirectionally selected sex-specific signal of offspring development in the alpine swift. Evolution 60:2370-2380.

Bize, P., A. Roulin, J. L. Tella, L.-F. Bersier, and H. Richner. 2004. Additive effects of ectoparasites over the reproductive attempts in the long-lived alpine swifts. Journal of Animal Ecology 73:1080-1088.

Bize, P., A. Roulin, J. L. Tella, and H. Richner. 2005. Femalebiased mortality in experimentally parasitized Alpine Swift Apus melba nestlings. Functional Ecology 19:405-413.

Blount, J. D., D. C. Houston, P. F. Surai, and A. P. Møller. 2004. Egg-laying capacity is limited by carotenoid pigment availability in wild gulls Larus fuscus. Proceedings of the Royal Society B (Supplement) 270:S79-S81.

Brief, S., and B. P. Chew. 1985. Effects of vitamin-A and betacarotene on reproductive performance in gilts. Journal of Animal Science 60:998-1004.

Broussard, D. R., T. S. Risch, F. S. Dobson, and J. O. Murie. 2003. Senescence and age-related reproduction of female Columbian ground squirrels. Journal of Animal Ecology 72: 212-219.

Brzezinska-Slebodzinska, E. 2001. Erythrocyte osmotic fragility test as the measure of defence against free radicals in rabbits of different age. Acta Veterinaria Hungarica 49:413-419.

Cadenas, E., and K. J. A. Davies. 2000. Mitochondrial free radical generation, oxidative stress, and aging. Free Radical Biology and Medicine 29:222-230.

Chew, B. P., and R. G. Archer. 1983. Comparative role of vitamin-A and beta-carotene on reproduction and neonate survival in rats. Theriogenology 20:459-472.

Criscuolo, F., M. D. Gonzalez-Barroso, F. Bouillaud, D. Ricquier, B. Miroux, and G. Sorci. 2005. Mitochondrial uncoupling proteins: new perspectives for evolutionary ecologists. American Naturalist 166:686-699.

Falnes, P. O., A. Klungland, and I. Alseth. 2007. Repair of methyl lesions in DNA and RNA by oxidative demethylation. Neuroscience 145:1222-1232.

Finkel, T., and N. J. Holbrook. 2000. Oxidants, oxidative stress and the biology of ageing. Nature 408:239-247.

George, S. B., J. M. Lawrence, A. L. Lawrence, J. Smiley, and L. Plank. 2001. Carotenoids in the adult diet enhance egg and juvenile production in the sea urchin Lytechinus variegatus. Aquaculture 199:353-369.

Gil, L., W. Siems, B. Mazurek, J. Gross, P. Schroeder, P. Voss, and T. Grune. 2006. Age-associated analysis of oxidative stress parameters in human plasma and erythrocytes. Free Radical Research 40:495-505.

Holzenberger, M., J. Dupont, B. Ducos, P. Leneuve, A. Geloen, P. C. Even, P. Cervera, and Y. Le Bouc. 2003. IGF-1 receptor regulates lifespan and resistance to oxidative stress in mice. Nature 421:182-187.

Ishii, N., M. Fujii, P. S. Hartman, M. Tsuda, K. Yasuda, N. Senoo-Matsuda, S. Yanase, D. Ayusawa, and K. Suzuki. 1998. A mutation in succinate dehydrogenase cytochrome b causes oxidative stress and ageing in nematodes. Nature 394:694-697.

Johnson, T. E. 2006. Recent results: biomarkers of aging. Experimental Gerontology 41:1243-1246.

Lesgards, J. F., P. Durand, M. Lassarre, P. Stocker, G. Lesgards, A. Lanteaume, M. Prost, and M. P. LehucherMichel. 2002. Assessment of lifestyle effects on the overall antioxidant capacity of healthy subjects. Environmental Health Perspectives 110:479-486.

Leslie, M. 2001. Aging research grows up. Science of Aging Knowledge Environment 2001(1):oa1. 〈http://sageke. sciencemag.org/cgi/content/full/2001/1/oal $\rangle$ 
Linton, S., M. J. Davies, and R. T. Dean. 2001. Protein oxidation and ageing. Experimental Gerontology 36:15031518.

McGraw, K. J., E. Adkins-Regan, and R. S. Parker. 2005. Maternally derived carotenoid pigments affect offspring survival, sex ratio, and sexual attractiveness in a colorful songbird. Naturwissenschaften 92:375-380.

McNamara, J. M., and A. I. Houston. 1996. State-dependent life histories. Nature 380:215-221.

Monaghan, P., and M. F. Haussmann. 2006. Do telomere dynamics link lifestyle and lifespan? Trends in Ecology and Evolution 21:47-53.

Moskovitz, J., S. Bar-Noy, W. M. Williams, B. S. Berlett, and E. R. Stadtman. 2001. Methionine sulfoxide reductase (MsrA) is a regulator of antioxidant defense and lifespan in mammals. Proceedings of the National Academy of Sciences (USA) 98:12920-12925.

Nilsson, J.-A. 2002. Metabolic consequences of hard work. Proceedings of the Royal Society B 269:1735-1739.

Pappas, A. C., T. Acamovic, N. H. C. Sparks, P. F. Surai, and R. M. McDevitt. 2006. Effects of supplementing broiler breeder diets with organoselenium compounds and polyunsaturated fatty acids on hatchability. Poultry Science 85 : 1584-1593.

Parkes, T. L., A. J. Elia, D. Dickinson, A. J. Hilliker, J. P. Phillips, and G. L. Boulianne. 1998. Extension of Drosophila lifespan by overexpression of human SOD1 in motorneurons. Nature Genetics 19:171-174.

Pettersson, A., and A. Lignell. 1999. Astaxanthin deficiency in eggs and fry of Baltic salmon (Salmo salar) with the M74 syndrome. Ambio 28:43-47.

Pike, T., J. D. Blount, B. Bjerken, J. Lindström, and N. B. Metcalfe. 2007. Carotenoids, oxidative stress and female mating preference for longer lived males. Proceedings of the Royal Society B 274:1591-1596.

Praticò, D. 2002. Lipid peroxidation and the aging process. Science of Aging Knowledge Environment 50:re5. 〈http:// sageke.sciencemag.org/cgi/content/abstract/2002/50/re5〉

Reid, J. M., E. M. Bignal, S. Bignal, D. I. McCracken, and P. Monaghan. 2003a. Environmental variability, life-history covariation and cohort effects in the red-billed chough
Pyrrhocorax pyrrhocorax. Journal of Animal Ecology 72: 36- 46.

Reid, J. M., E. M. Bignal, S. Bignal, D. I. McCracken, and P. Monaghan. 2003b. Age-specific reproductive performance in red-billed choughs Pyrrhocorax pyrrhocorax: patterns and processes in a natural population. Journal of Animal Ecology 72:765-776.

Ricklefs, R. E., and M. Wikelski. 2002. The physiology/lifehistory nexus. Trends in Ecology and Evolution 17:462-468.

Ruan, H., X. D. Tang, M. L. Chen, M. A. Joiner, G. Sun, N. Brot, H. Weissbach, S. H. Heinemann, L. Iverson, C. F. Wu, and T. Hoshi. 2002. High-quality life extension by the enzyme peptide methionine sulfoxide reductase. Proceedings of the National Academy of Sciences (USA) 99:2748-2753.

Sahin, N., K. Sahin, M. Onderci, M. Karatepe, M. O. Smith, and O. Kucuk. 2006. Effects of dietary lycopene and vitamin E on egg production, antioxidant status and cholesterol levels in Japanese quail. Asian-Australasian Journal of Animal Sciences 19:224-230.

Shahidi, F., Metusalach, and J. A. Brown. 1998. Carotenoid pigments in seafoods and aquaculture. Critical Reviews in Food Science and Nutrition 38:1-67.

Stearns, S. C. 1992. The evolution of life histories. Oxford University Press, Oxford, UK.

van de Pol, M., and S. Verhulst. 2006. Age-dependent traits: a new statistical method to separate within- and betweenindividual effects. American Naturalist 167:766-773.

Van Noordwijk, A. J., and G. de Jong. 1986. Acquisition and allocation of resources: their influence on variation in life history tactics. American Naturalist 128:137-142.

Weladji, R. B., J. M. Gaillard, N. G. Yoccoz, O. Holand, A. Mysterud, A. Loison, M. Nieminen, and N. C. Stenseth. 2006. Good reindeer mothers live longer and become better in raising offspring. Proceedings of the Royal Society B 273: $1239-1244$

Wiersma, P., C. Selman, J. R. Speakman, and S. Verhulst. 2004. Birds sacrifice oxidative protection for reproduction. Proceedings of the Royal Society B 270(Supplement):S360-S363.

Zera, A. J., and L. G. Harshman. 2001. The physiology of life history trade-offs in animals. Annual Review of Ecology and Systematics 32:95-126. 